\title{
Numerical Analysis of Waveguide-Based Surface Plasmon Resonance Sensor with Adsorbed Layer Using Two- and Three-Dimensional Beam-Propagation Methods
}

\author{
Jun SHIBAYAMA $^{\dagger \mathrm{a})}$, Member, Shota TAKAGI ${ }^{\dagger}$, Tomohide YAMAZAKI ${ }^{\dagger}$, Student Members, \\ Junji YAMAUCHI ${ }^{\dagger}$, Member, and Hisamatsu NAKANO ${ }^{\dagger}$, Fellow
}

\begin{abstract}
SUMMARY A waveguide-based surface plasmon resonance (SPR) sensor with an adsorbed layer is analyzed using the beam-propagation method. For two-dimensional (2-D) models, numerical results show that the change in thickness of the adsorbed layer placed on the metal leads to a significant shift of the maximum absorption wavelength. Through eigenmode analysis, the maximum absorption wavelength is found to be consistent with the cutoff wavelength of the second-order surface plasmon mode. The designed 2-D sensor shows an absorption wavelength shift from 0.595 to $0.603 \mu \mathrm{m}$, when the analyte refractive index is increased from 1.330 to 1.334. After a basic investigation using the 2-D models, we next study 3-D models. When the metal with the absorbed layer is wide enough to cover the core region, the 3 -D results are similar to the 2-D results. However, as the metal width is reduced, the absorption wavelength shifts toward a shorter wavelength and the sensitivity to the refractive index change degrades gradually. The degradation of the sensitivity is considerable when the metal width is narrower than the core width. As a result, the metal width of the practical SPR sensor should be slightly wider than the core width so as to maintain the sensitivity corresponding to that obtained for the 2-D model.

key words: surface plasmon resonance (SPR), optical sensor, optical waveguide, beam-propagation method (BPM), imaginary-distance procedure
\end{abstract}

\section{Introduction}

The Kretschmann configuration, composed of a prism coated with a thin metal film, has widely been employed for optical sensors using surface plasmon resonance (SPR) coupling [1], [2]. In this configuration, the value of the refractive index can be found from the reflection minima of a surface plasma wave at a given frequency, allowing realtime and high-resolution measurements. For this configuration, however, monolithic integration into optical circuits is generally difficult.

To integrate an SPR sensor into optical circuits, waveguide-based SPR sensors have been investigated [1][9]. Theoretically, the sensitivity of waveguide-based SPR sensors is approximately the same as that of the Kretschmann configuration [1]. Previous studies have involved the design of several waveguide-based SPR sensors using analytical or numerical methods. For example, an SPR sensor with an adsorbed layer has been designed to

Manuscript received May 31, 2006.

Manuscript revised September 19, 2006.

${ }^{\dagger}$ The authors are with the Faculty of Engineering, Hosei University, Koganei-shi, 184-8584 Japan.

a)E-mail: shiba@k.hosei.ac.jp

DOI: 10.1093/ietele/e90-c.1.95 shift the peak wavelength of SPR coupling [4]-[6]. However, detailed eigenmode analysis has not been performed for explaining the mechanism of the peak wavelength shift with an adsorbed layer. In addition, all the theoretical investigations have been restricted to two-dimensional (2-D) models and practical three-dimensional (3-D) models have not yet been treated.

In this study, we analyze waveguide-based SPR sensors by both 2-D and 3-D beam-propagation methods (BPMs) [10] and discuss sensing characteristics for aqueous environments. First, we present brief explanations of the BPMs for propagating beam analysis [10], [11] and the imaginarydistance (ID) BPM for eigenmode analysis [12]-[19]. Next, we investigate the effect of metal thickness on the sensing characteristics for the 2-D model. It is shown that the maximum absorption wavelength slightly shifts to a longer wavelength as the metal thickness increases. After choosing the metal thickness, we study the case where the adsorbed layer is placed on the metal layer. The change in adsorbed layer thickness also leads to a shift of the maximum absorption wavelength. The mechanism of this wavelength shift is explained in detail through eigenmode analysis using the IDBPM. The designed SPR sensor shows the absorption wavelength shift from 0.594 to $0.602 \mu \mathrm{m}$, when the refractive index of the analyte is increased from 1.330 to 1.334 , which is detectable by an optical spectrum analyzer.

Finally, we analyze the 3-D models, paying attention to the dependence of metal width on sensing characteristics. When the metal with the adsorbed layer is wide enough to cover the core region, the 3 -D results are similar to the $2-\mathrm{D}$ results. It is revealed that for a narrow metal width of $3 \mu \mathrm{m}$, which is slightly wider than the core size ( $2 \mu \mathrm{m}$ wide), the absorption wavelength shifts towards a shorter wavelength than that in the 2-D results, while maintaining sensitivity to the refractive index change. However, a further reduction in metal width gives rise to a reduced sensitivity. As a result, the 3-D analysis provides important information on the choice of metal width, which affects the sensitivity to the refractive index change.

\section{Numerical Methods}

2-D models of SPR sensors have been analyzed by semianalytic techniques such as the transfer matrix method (TMM) 
[20]. The use of the TMM provides efficient eigenmode calculations of dispersion characteristics. Unfortunately, 3-D models cannot be treated by the TMM. In addition, one must resort to other numerical techniques when the propagating beam in the SPR sensor is analyzed. In contrast, the BPM has the advantage that eigenmode analysis with the ID procedure followed by propagating beam analysis can be performed using a single BPM algorithm. More importantly, the BPM can treat 3-D models of SPR sensors. These facts motivate us to apply the BPM to the analysis of SPR sensors.

Here, we provide brief explanations of the BPM [10], [11]. The basic equation for the 3-D semivectorial BPM is expressed as

$$
\frac{\partial \phi}{\partial z}=-\frac{j}{2 k n_{0}}\left[D_{x x}+D_{y y}+k^{2}\left(n^{2}-n_{0}^{2}\right)\right] \phi,
$$

where

$$
D_{x x}=n^{2} \frac{\partial}{\partial x}\left(\frac{1}{n^{2}} \frac{\partial}{\partial x}\right), D_{y y}=\frac{\partial^{2}}{\partial y^{2}}, \phi=H_{y}
$$

for the TE mode and

$$
D_{x x}=\frac{\partial^{2}}{\partial x^{2}}, D_{y y}=n^{2} \frac{\partial}{\partial y}\left(\frac{1}{n^{2}} \frac{\partial}{\partial y}\right), \phi=H_{x}
$$

for the TM mode, in which $k$ is the free-space wavenumber, $n$ is the refractive index, and $n_{0}$ is the reference refractive index to be appropriately chosen. After approximating the spatial derivatives using a modified finite-difference formula [10], we solve Eq. (1) using the Crank-Nicolson scheme. Using the BPM, we can determine how the input field propagates in optical waveguides [11].

The BPM can also be used as an eigenmode solver with the ID procedure [12]-[19]. An arbitrary input field can be represented as a summation of the eigenmodes in the form

$$
\phi(x, y, z=0)=\sum a_{i} \phi_{i}(x, y),
$$

where $\phi_{i}$ and $a_{i}$ represent the $i$ th eigenmode field and its amplitude, respectively. At a distance $z$, the solution of Eq. (1) can be written as

$$
\phi(x, y, z)=\sum a_{i} e^{-j \lambda_{i} z} \phi_{i}(x, y)
$$

where $\lambda_{i}=\beta_{i}-k n_{0}$, in which $\beta_{i}$ is the propagation constant of the $i$ th eigenmode. Here, we change the propagation axis to an imaginary axis, i.e., $z=j \tau$, leading to

$$
\phi(x, y, \tau)=\sum a_{i} e^{\lambda_{i} \tau} \phi_{i}(x, y) .
$$

As can be seen from Eqs. (3) and (4), in the ID procedure, the sinusoidal phase change of the field is replaced with its exponential amplitude change. The amplitude of the $i$ th eigenmode field is determined by the following amplification factor $A F_{i}$ :

$$
\phi_{i}^{l+1}=\frac{1+\frac{\Delta \tau k^{2}}{4 k n_{0}}\left(\left(\beta_{i} / k\right)^{2}-n_{0}^{2}\right)}{1-\frac{\Delta \tau k^{2}}{4 k n_{0}}\left(\left(\beta_{i} / k\right)^{2}-n_{0}^{2}\right)} \phi_{i}^{l} \equiv A F_{i} \phi_{i}^{l} .
$$

Using appropriate parameters makes the amplitude of a specific mode quite large, when compared with those of other modes. Therefore, only the desired mode is generated efficiently.

Once the eigenmode field is obtained, the propagation constant is readily calculated on the basis of the following variational expression without using the weak form:

$$
(\beta / k)^{2}=\frac{\iint\left[k^{2} n^{2}|\phi|^{2}-\phi^{*}\left(D_{x x} \phi+D_{y y} \phi\right)\right] d x d y}{\iint|\phi|^{2} d x d y},
$$

where the superscript $*$ represents the complex conjugate. The real and imaginary parts of Eq. (6) correspond to the phase and attenuation constants, respectively.

\section{Analysis of Waveguide-Based SPR Sensors}

\subsection{Analysis of 2-D Model}

We start with the analysis of the 2-D waveguide-based SPR sensor shown in Fig. 1. The SPR sensors treated here are supposed to operate around $\lambda=0.6 \mu \mathrm{m}$, which corresponds to the center wavelength of a tunable laser. Water is chosen as an analyte. We excite the field of the fundamental TM mode in the input waveguide. Varying the operating wavelength, we evaluate the output power from the waveguide, which depends on the refractive index of the analyte $n_{\mathrm{a}}$. The metal is chosen to be Au and the dispersion property of its refractive index is taken into account using the Drude model dielectric function as

$$
n_{\mathrm{m}}^{2}=1+\frac{\omega_{\mathrm{p}}^{2}}{\omega(j v-\omega)},
$$

where $\omega$ is the angular frequency, $\omega_{\mathrm{p}}$ is the electron plasma frequency, and $v$ is the effective electron collision frequency. These values are determined using $n_{\mathrm{m}}=0.131-j 3.645$ at $0.6328 \mu \mathrm{m}$ [21], i.e., $\omega_{\mathrm{p}}=1.127 \times 10^{16} \mathrm{rad} / \mathrm{sec}$ and $v=$

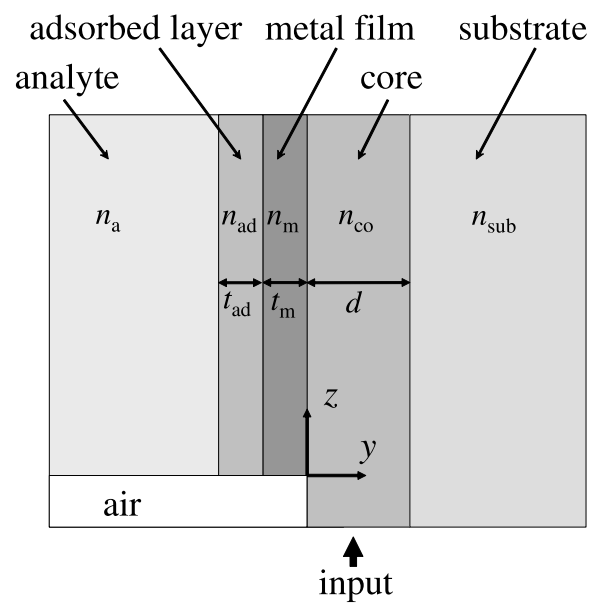

Fig. 1 Configuration of waveguide-based SPR sensor $(d=2 \mu \mathrm{m})$. The refractive indices are $n_{\mathrm{co}}=n_{\mathrm{ad}}=1.47, n_{\mathrm{sub}}=1.46$ [4], and $n_{\mathrm{m}}=0.131-$ $j 3.645$ at $0.6328 \mu \mathrm{m}[21]$. The analyte is thick enough to give converged solutions of the numerical results. 


\section{$1.992 \times 10^{14} \mathrm{rad} / \mathrm{sec}$}

First, we investigate the sensing characteristics for a different metal thickness $t_{\mathrm{m}}$ without the adsorbed layer $\left(t_{\mathrm{ad}}=\right.$ 0 ). Figure 2 shows the output power normalized to the input power as a function of wavelength, calculated using the BPM. The refractive index of the analyte is $n_{\mathrm{a}}=1.332$. The device length is chosen to be $200 \mu \mathrm{m}$. The numerical parameters are as follows: the transverse sampling width $\Delta x=0.001 \mu \mathrm{m}$ and the longitudinal sampling width $\Delta z=$ $0.01 \mu \mathrm{m}$.

It is seen in Fig. 2 that as the metal becomes thick, the maximum absorption wavelength slightly shifts to a longer wavelength, reaching a limit value around $0.56 \mu \mathrm{m}$. In addition, a thick metal reduces the coupling between the waveguide and surface plasmon (SP) modes. Here, we choose $t_{\mathrm{m}}=0.045 \mu \mathrm{m}$, which allows a relatively strong coupling between the waveguide and SP modes.

One technique for shifting the maximum absorption wavelength is to add an adsorbed layer to the metal layer [4]-[6], as shown in Fig. 1. Figure 3 demonstrates the wavelength shift when the adsorbed layer is added. It is

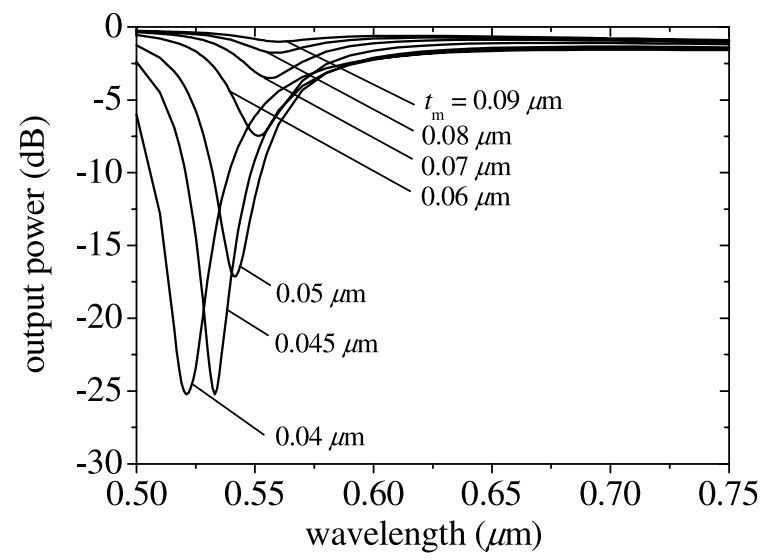

Fig. 2 Output power against wavelength. The metal thickness ranges from $t_{\mathrm{m}}=0.04 \mu \mathrm{m}$ to $0.09 \mu \mathrm{m}$. The refractive index of the analyte is $n_{\mathrm{a}}=$ 1.332 .

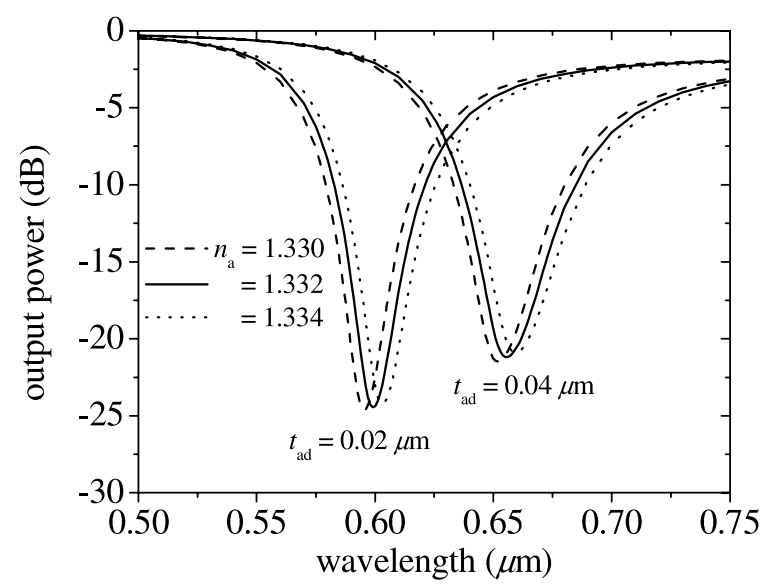

Fig. 3 Output power against wavelength. The metal thickness is $t_{\mathrm{m}}=$ $0.045 \mu \mathrm{m}$. The adsorbed layer thickness is $t_{\mathrm{ad}}=0.02 \mu \mathrm{m}$ or $t_{\mathrm{ad}}=0.04 \mu \mathrm{m}$. found that the maximum absorption wavelengths are around $0.6 \mu \mathrm{m}$ and $0.66 \mu \mathrm{m}$ for $t_{\mathrm{ad}}=0.02 \mu \mathrm{m}$ and $t_{\mathrm{ad}}=0.04 \mu \mathrm{m}$, respectively. Figure 3 also shows that the absorption wavelength depends on the refractive index of the analyte, $n_{\mathrm{a}}$. For example, the sensor with $t_{\mathrm{ad}}=0.02 \mu \mathrm{m}$ offers the absorption wavelength shift from 0.595 to $0.603 \mu \mathrm{m}$, when the refractive index of the analyte is increased from 1.330 to 1.334 , which is detectable by an optical spectrum analyzer.

As discussed above, the addition of the adsorbed layer has been effective in shifting the absorption wavelength. It should be noted, however, that a detailed explanation for the wavelength shift has not been given. Therefore, we next investigate the effect of the adsorbed layer on the dispersion characteristics by eigenmode analysis with the ID-BPM.

The dispersion characteristics for $t_{\mathrm{ad}}=0.02 \mu \mathrm{m}$ and $t_{\mathrm{ad}}=0.04 \mu \mathrm{m}$ are given in Figs. 4 and 5, respectively, in which (a) and (b) indicate the results for the effective index and loss, respectively. In this analysis, $n_{\mathrm{a}}=1.332$ is used. In (a), also included is the effective index of the TM waveguide mode of the input waveguide $(z<0$ in Fig. 1). In Figs. 4 and 5 , there exist two SP modes in this wavelength range. The second mode has the cutoff wavelength: $\lambda \simeq 0.6 \mu \mathrm{m}$ for $t_{\mathrm{ad}}=0.02 \mu \mathrm{m}$ and $\lambda \simeq 0.66 \mu \mathrm{m}$ for $t_{\mathrm{ad}}=0.04 \mu \mathrm{m}$.

It can be seen in Fig. 4(a) that at $\lambda \simeq 0.595 \mu \mathrm{m}$, the effective index difference between the waveguide mode and

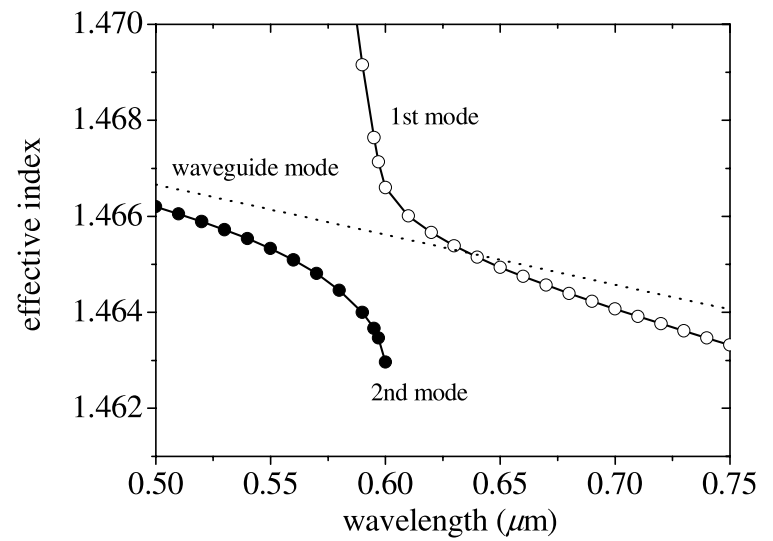

(a)

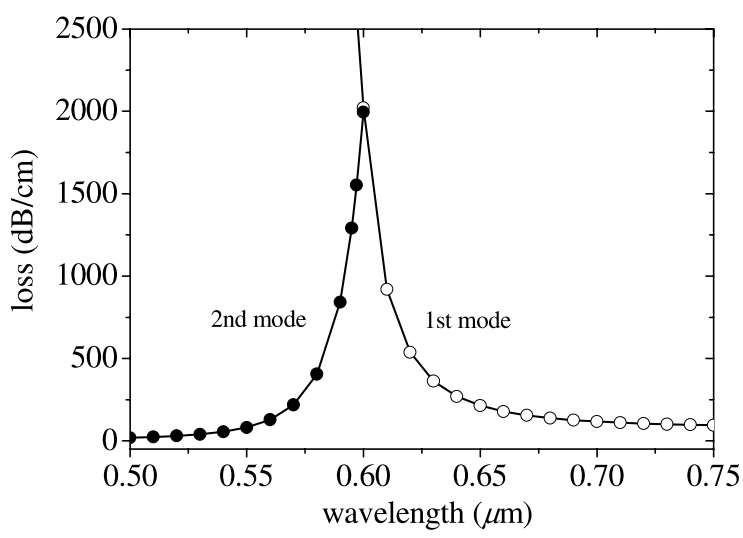

(b)

Fig. 4 Effective index (a) and loss (b) as functions of wavelength $\left(t_{\text {ad }}=\right.$ $0.02 \mu \mathrm{m}$ ). 


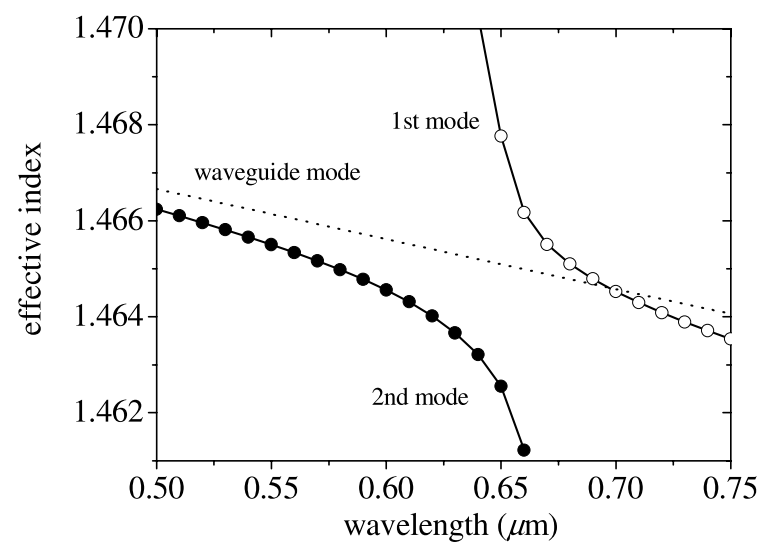

(a)

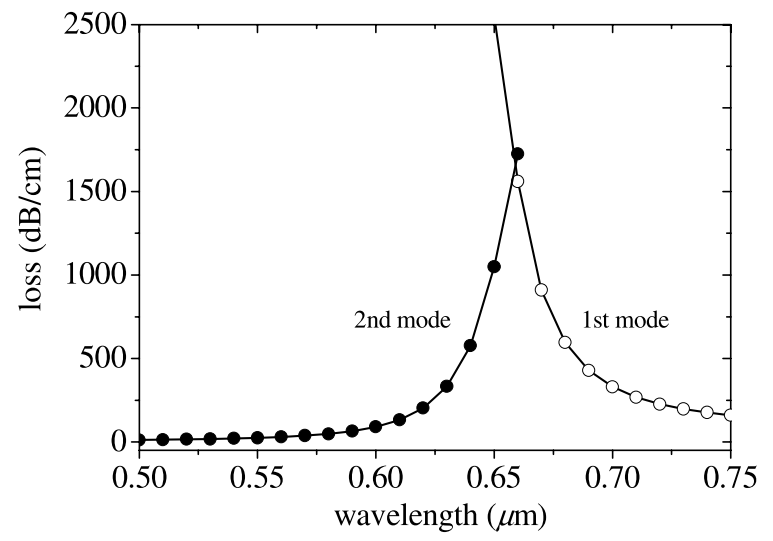

(b)

Fig. 5 Effective index (a) and loss (b) as functions of wavelength $\left(t_{\mathrm{ad}}=\right.$ $0.04 \mu \mathrm{m})$.

the first mode is almost the same as that between the waveguide mode and the second mode. For operating wavelengths shorter than $0.595 \mu \mathrm{m}$, the effective index of the waveguide mode is close to that of the second mode. Therefore, the waveguide mode is coupled mainly to the second mode. On the other hand, for wavelengths longer than $0.595 \mu \mathrm{m}$, the second mode becomes cut off and the effective index of the waveguide mode is close to that of the first mode. It follows that the waveguide mode is coupled to the first mode. Notice that at $\lambda \simeq 0.595 \mu \mathrm{m}$, the propagation loss becomes almost maximal in Fig. 4(b). Therefore, at this wavelength (close to the cutoff wavelength of the second mode) the SPR coupling becomes strong. This explains the maximum absorption wavelength found in Fig. 3.

The above discussion holds true for the case of $t_{\mathrm{ad}}=$ $0.04 \mu \mathrm{m}$ in Fig. 5, in which the absorption wavelength shifts to $\lambda \simeq 0.66 \mu \mathrm{m}$. As a result, the change in adsorbed layer thickness leads to a shift of the cutoff wavelength of the second SP mode, which is consistent with the maximum absorption wavelength of the SPR sensors.

Typical field distributions $\left(t_{\mathrm{ad}}=0.02 \mu \mathrm{m}\right)$ are shown in Figs. 6(a) and (b) for the first and second modes, respectively. Each field is normalized to its maximum amplitude. It is seen that for the second mode, the local minimum of the field exists in the core region. At $\lambda=0.6 \mu \mathrm{m}$, the field

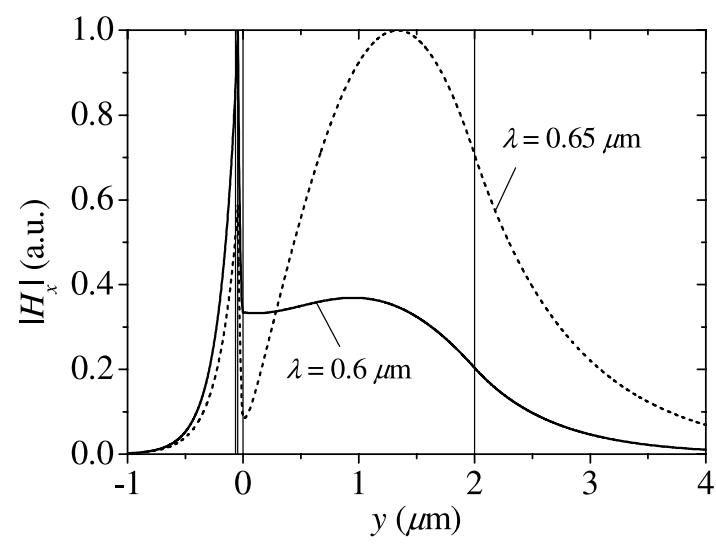

(a)

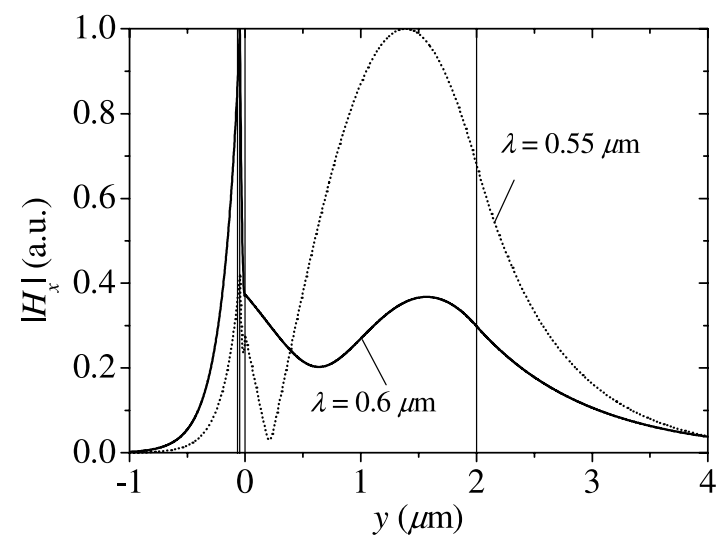

(b)

Fig. 6 Field distributions for $t_{\mathrm{ad}}=0.02 \mu \mathrm{m}$. First mode (a) and second mode (b).

amplitude is significantly large in the metal region both for the first and second modes. This is why the loss becomes maximal at $\lambda=0.6 \mu \mathrm{m}$, as observed in Fig. 4(b).

\subsection{Analysis of 3-D Model}

We now analyze the 3-D SPR sensor shown in Fig. 7 (note that the 2-D model treated above corresponds to the case where $w=d_{w}=\infty$ in Fig. 7). A square core with $d=$ $d_{w}=2 \mu \mathrm{m}$ is used and the metal strip is centered on the core. Other configuration parameters are the same as those used in the 2-D model.

Figure 8 shows the output power at $z=200 \mu \mathrm{m}$ for $w=$ $10 \mu \mathrm{m}$. It can be seen that the wavelength responses of the 3-D SPR sensor are similar to those of the 2-D SPR sensor (Fig. 3). The sensor with $t_{\mathrm{ad}}=0.02 \mu \mathrm{m}$ is found to offer an absorption wavelength shift from 0.598 to $0.606 \mu \mathrm{m}$, when the refractive index of the analyte is increased from 1.330 to 1.334 . This sensitivity to the refractive index change is almost the same as that for the 2-D sensor (Fig. 3). The 2$\mathrm{D}$ analysis, therefore, can efficiently provide a reasonable estimation of the sensitivity of the 3 -D sensor with the metal being wide enough to cover the core region.

Note that the maximum absorption for the 3-D sensor (Fig. 8) is less than that for the 2-D sensor (Fig. 3) by about 


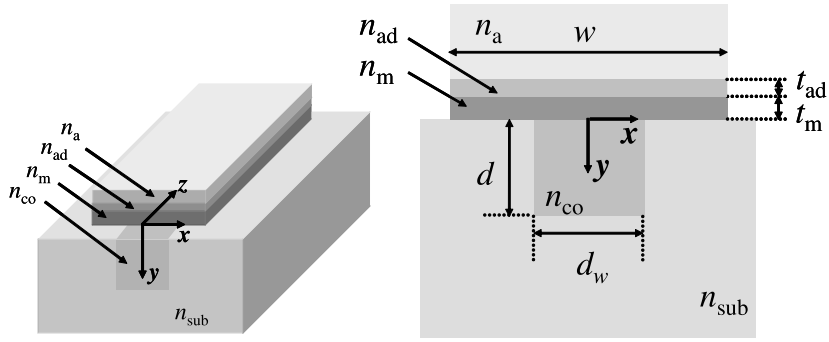

Fig. 7 Configuration of 3-D waveguide-based SPR sensor. The right figure shows the $x-y$ cross section of the sensor.

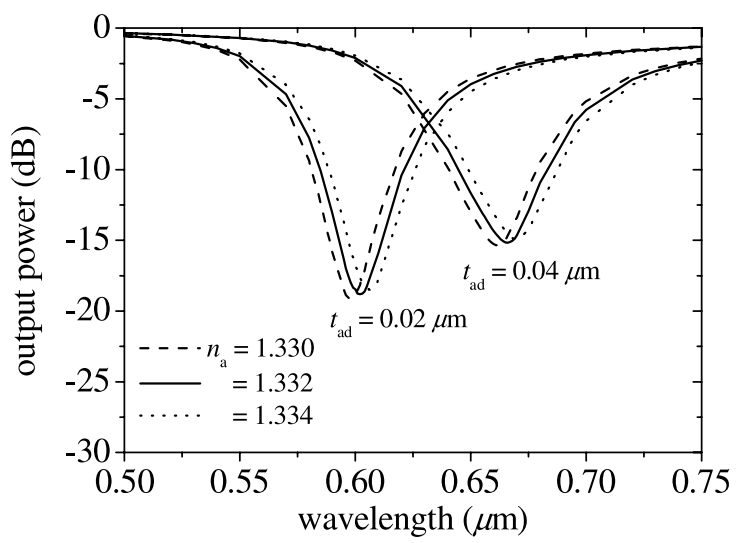

Fig. 8 Output power against wavelength for $w=10 \mu \mathrm{m}$.

$5 \mathrm{~dB}$, even though the sensor lengths are the same for both models $(200 \mu \mathrm{m})$. This is because the amplitude of the mode field along the metal surface for the 3-D sensor gradually decreases, as the transverse position $x$ increases in distance away from the center of the waveguide ( $x=0$ in Fig. 7). The decrease in field amplitude reduces the absorption of the field by the metal. This effect cannot be taken into account in the 2-D analysis, since the amplitude of the mode field is uniform in the $x$ direction due to $w=d_{w}=\infty$. These facts indicate that the 2-D analysis provides a slight overestimation of the value of the maximum absorption.

The dependence of the width $w$ on the sensing characteristics, which cannot be treated using the 2-D analysis, is further investigated in Fig. 9, in which $n_{\mathrm{a}}=1.332$ and $t_{\mathrm{ad}}=0.02 \mu \mathrm{m}$ are used. This investigation is of importance since a reduction in $w$ leads to the reduced amount of the analyte. In Fig. 9, it is found that as $w$ becomes narrow, the absorption wavelength shifts toward a shorter wavelength (although not illustrated, the wavelength response down to $w=5 \mu \mathrm{m}$ is almost the same as that for $w=10 \mu \mathrm{m}$ ).

We further evaluate the sensitivity to the refractive index change. For $w=10 \mu \mathrm{m}$, the absorption wavelength shift is $8 \mathrm{~nm}$, when the analyte refractive index changes from 1.330 to 1.334 (as shown in Fig. 8). This sensitivity is maintained for the case down to $w=3 \mu \mathrm{m}$, which is slightly wider than the core size. It should be noted, however, that the sensitivity decreases when $w$ is comparable to or less than the core width: for $w=2$ and $1 \mu \mathrm{m}$, the wavelength shifts are 7 and $6 \mathrm{~nm}$, respectively. Therefore, the metal

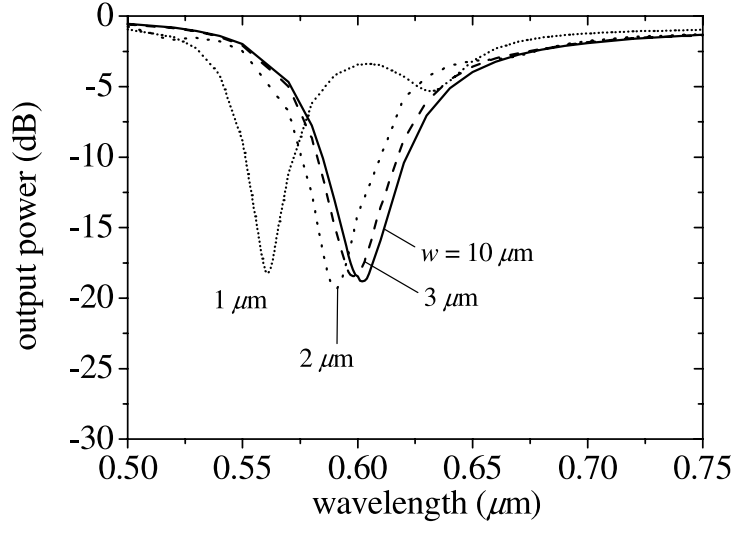

Fig.9 Output power against wavelength for several metal widths.

width should be slightly wider than the core width so that a reasonable sensitivity may be maintained.

\section{Conclusion}

We analyzed 2-D and 3-D SPR sensors using BPMs. For the 2-D models, the effect of the adsorbed layer on the dispersion characteristics was discussed in detail through eigenmode analysis with the ID-BPM. It was shown that a change in adsorbed layer thickness leads to a shift of the cutoff wavelength of the second-order mode. This wavelength was found to be consistent with the maximum absorption wavelength of the SPR sensors. We further investigated the 3-D models, paying attention to the dependence of metal width on sensing characteristics. When the metal width is wide enough to cover the core region, the sensitivity to refractive index change for the 3-D model is similar to those for the 2-D models. However, when the metal width is comparable to or less than the core width, the characteristics of the 3-D models differ from those of the 2-D models. In particular, the 3-D SPR sensor exhibits a reduced sensitivity compared with that of the 2-D sensor. As a result, the metal width of the practical SPR sensor should be slightly wider than the core width so as to maintain the sensitivity corresponding to that obtained for the 2-D model.

\section{Acknowledgment}

This work was supported in part by the Takahashi Industrial and Economic Research Foundation.

\section{References}

[1] J. Homola, S.S. Yee, and G. Gauglitz, "Surface plasmon resonance sensors: Review," Sens. Actuators B, vol.54, pp.3-15, Jan. 1999.

[2] J. Homola, "Present and future of surface plasmon resonance biosensors," Analytical and Bioanalytical Chemistry, vol.377, no.3, pp.528-539, Oct. 2003.

[3] L.M. Lechuga, "Optical sensors based on evanescent field sensing Part II, Integrated optical sensors," Quimica Analitica, vol.19, pp.61-67, 2000.

[4] R.D. Harris and J.S. Wilkinson, "Waveguide surface plasmon resonance sensors," Sens. Actuators B, vol.29, pp.261-267, Oct. 1995. 
[5] M.N. Weiss, R. Srivastava, H. Groger, P. Lo, and S.F. Luo, "A theoretical investigation of environmental monitoring using surface plasmon resonance waveguide sensors," Sens. Actuators A, vol.51, pp.211-217, Nov. 1996.

[6] J. Čtyroký, J. Homola, and M. Skalsky, "Tuning of spectral operation range of a waveguide surface plasmon resonance sensor," Electron. Lett., vol.33, no.14, pp.1246-1248, July 1997.

[7] J. Čtyroký, J. Homola, P.V. Lambeck, S. Musa, H.J.W.M. Hoekstra, R.D. Harris, J.S. Wilkinson, B. Usievich, and N.M. Lyndin, "Theory and modelling of optical waveguide sensors utilising surface plasmon resonance," Sens. Actuators B, vol.54, pp.66-73, Jan. 1999.

[8] C.R. Lavers, K. Itoh, S.C. Wu, M. Murabayashi, I. Mauchline, G. Stewart, and T. Stout, "Planar optical waveguides for sensing applications," Sens. Actuators B, vol.69, pp.85-95, Sept. 2000.

[9] J. Dostalek, J. Čtyroký, J. Homola, E. Brynda, M. Skalsky, P Nekvindova, J. Spirkova, J. Skvor, and J. Schrofel, "Surface plasmon resonance biosensor based on integrated optical waveguide," Sens. Actuators B, vol.76, pp.8-12, June 2001.

[10] J. Yamauchi, Propagating Beam Analysis of Optical Waveguides, Research Studies Press, Baldock, Hertfordshire, U.K., 2003.

[11] T. Yamazaki, H. Aono, J. Yamauchi, J. Shibayama, and H. Nakano, "Propagation loss of bent light-guiding metal line loaded on embedded dielectric core," Electron. Lett., vol.41, no.6, pp.319-320, March 2005

[12] D. Yevick and B. Hermansson, "New formulations of the matrix beam propagation method: Application to rib waveguides," IEEE J. Quantum Electron., vol.25, no.2, pp.221-229, Feb. 1989.

[13] C.L. Xu, W.P. Huang, and S.K. Chaudhuri, "Efficient and accurate vector mode calculations by beam propagation method," J. Lightwave Technol., vol.11, no.7, pp.1209-1215, July 1993.

[14] S. Jüngling and J.C. Chen, "A study and optimization of eigenmode calculations using the imaginary-distance beam-propagation method," IEEE J. Quantum Electron., vol.30, no.9, pp.2098-2105, Sept. 1994

[15] M.M. Spühler, D. Wiesmann, P. Freuler, and M. Diergardt, "Direct computation of higher-order propagation modes using the imaginary-distance beam propagation method," Optical Quantum Electron., vol.31, no.9-10, pp.751-761, Oct. 1999.

[16] K. Saitoh and M. Koshiba, "Full-vectorial imaginary-distance beam propagation method based on a finite element scheme: Application to photonic crystal fibers," IEEE J. Quantum Electoron., vol.38, no.7, pp.927-933, July 2002.

[17] S.S.A. Obayya, B.M.A. Rahman, K.T.V. Grattan, and H.A. ElMikati, "Full vectorial finite-element-based imaginary distance beam propagation solution of complex modes in optical waveguides," J. Lightwave Technol., vol.20, no.6, pp.1054-1059, June 2002.

[18] P. Chamorro-Posada, "A modified imaginary distance BPM for directly computing arbitrary vector modes of 3-D optical waveguides,' J. Lightwave Technol., vol.21, no.3, pp.862-867, March 2003.

[19] J. Shibayama, T. Yamazaki, J. Yamauchi, and H. Nakano, "Eigenmode analysis of a light-guiding metal line loaded on a dielectric substrate using the imaginary-distance beam-propagation method," J. Lightwave Technol., vol.23, no.3, pp.1533-1539, March 2005.

[20] J. Chilwell and I. Hodgkinson, "Thin-films field-transfer matrix theory of planar multilayer waveguides and reflection from prismloaded waveguides," J. Opt. Soc. Am. A,, vol.1, no.7, pp.742-753, July 1984.

[21] Ş.K. Özdemir and G. Turhan-Sayan, "Temperature effects on surface plasmon resonance: Design considerations for an optical temperature sensor," J. Lightwave Technol., vol.21, no.3, pp.805-814, March 2003.

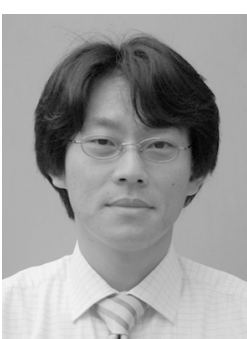

Jun Shibayama was born in Kashiwa, Japan, on July 1, 1969. He received his B.E. M.E., and Dr.E. degrees from Hosei University, Tokyo, Japan, in 1993, 1995, and 2001, respectively. In 1995, he joined Opto-Technology Laboratory (currently FITEL Photonics Laboratory), Furukawa Electric Co., Ltd., Ichihara, Chiba, Japan. Since 1999, he has been an assistant at Hosei University. His research interests include the numerical analysis of optical waveguides. Dr. Shibayama is a member of IEEE-

LEOS and the Optical Society of America.

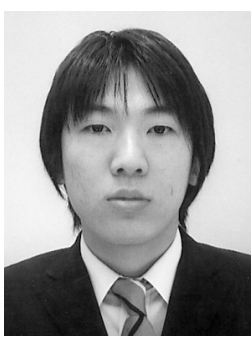

Shota Takagi was born in Kanagawa, Japan, on August 9, 1982. He received his B.E. degree from Hosei University, Tokyo, Japan, in 2005. $\mathrm{He}$ is currently pursuing an M.E. degree.

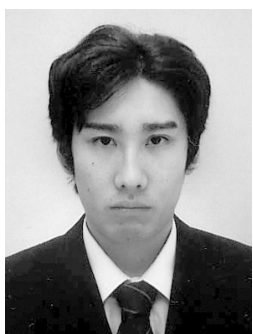

Tomohide Yamazaki was born in Tokyo, Japan, on May 1, 1979. He received his B.E. and M.E. degrees from Hosei University, Tokyo, Japan, in 2003 and 2005. He is currently pursuing a Dr.E. degree. Since 2005, he has been a Research Fellow of the Japan Society for the Promotion of Science. Mr. Yamazaki is a student member of the IEEE.

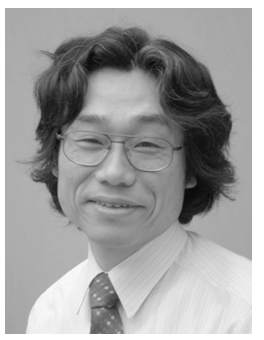

Junji Yamauchi was born in Nagoya, Japan, on August 23, 1953. He received his B.E., M.E., and Dr.E. degrees from Hosei University, Tokyo, Japan, in 1976, 1978, and 1982, respectively. From 1984 to 1988 , he served as a Lecturer in the Electrical Engineering Department of Tokyo Metropolitan Technical College. Since 1988 , he has been a member of the faculty of Hosei University, where he is now a Professor of Electronic Informatics. His research interests include optical waveguides and circularly polarized antennas. He is the author of Propagating Beam Analysis of Optical Waveguides (Baldock, Hertfordshire, U.K.: Research Studies Press, 2003). Dr. Yamauchi is a member of the IEEE and the Optical Society of America. 


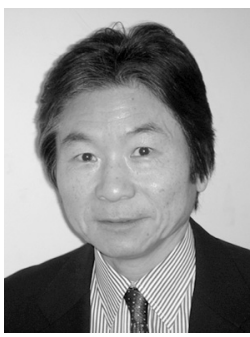

Hisamatsu Nakano was born in Ibaraki, Japan, on April 13, 1945. He received his B.E., M.E., and Dr.E. degrees in electrical engineering from Hosei University, Tokyo in 1968, 1970, and 1974, respectively. Since 1973, he has been a member of the faculty of Hosei University, where he is now a Professor of Electronic Informatics. His research interests include numerical methods for low- and high-frequency antennas and optical waveguides. He has published more than 200 refereed journal papers, more than 200 international symposium papers, and more than 750 national symposium papers. He is the author of a book entitled Helical and Spiral Antennas (New York: Research Studies Press, Wiley, 1987) and the coauthor of Analysis Methods of Electromagnetic Wave Problems, Volume Two (Norwood, MA: Artech House, 1986). In addition, he is the author of Helical and Spiral Antennas, Encyclopedia of Communications (New York: Wiley, 2002). $\mathrm{He}$ received the IEE International Conference on Antennas and Propagation Best Paper Award and the IEEE Transactions on Antennas and Propagation Best Application Paper Award (H. A. Wheeler Award) in 1989 and 1994, respectively. In 1992, he was elected an IEEE fellow for contributions to the design of spiral and helical antennas. In 2001, he received the Award of Distinguished Technical Communication (from the Society for Technical Communication, USA) and the Science and Technology Progress Award (from Hangzhou, China). Prof. Nakano is an associate editor of several journals and magazines, such as Electromagnetics, IEEE Antennas and Propagation Magazine, IEEE Antennas and Wireless Propagation Letters, and Asian Information-Science-Life. 